\title{
The Frobenius number in the set of numerical semigroups with fixed multiplicity and genus
}

\author{
Aureliano M. Robles-Pérez*† and José Carlos Rosales*ł
}

\begin{abstract}
We compute all possible numbers that are the Frobenius number of a numerical semigroup when multiplicity and genus are fixed. Moreover, we construct explicitly numerical semigroups in each case.
\end{abstract}

Keywords: Numerical semigroups, Frobenius number, multiplicity, genus.

2010 AMS Classification: 11D07, 20M14

\section{Introduction}

Let $\mathbb{Z}$ and $\mathbb{N}$ be the set of integers and the set of nonnegative integers respectively. A numerical semigroup is a subset $S$ of $\mathbb{N}$ that is closed under addition, $0 \in S$ and $\mathbb{N} \backslash S$ is finite.

If $A \subseteq \mathbb{N}$ is a nonempty subset of $\mathbb{N}$, then we denote by $\langle A\rangle$ the submonoid of $(\mathbb{N},+)$ generated by $A$, that is,

$$
\langle A\rangle=\left\{\lambda_{1} a_{1}+\ldots+\lambda_{n} a_{n} \mid n \in \mathbb{N} \backslash\{0\}, a_{1}, \ldots, a_{n} \in A, \text { and } \lambda_{1}, \ldots, \lambda_{n} \in \mathbb{N}\right\} .
$$

It is well known (see for instance [24]) that $\langle A\rangle$ is a numerical semigroup if and only if $\operatorname{gcd}(A)=1$, where $\operatorname{gcd}$ means greatest common divisor.

If $S$ is a numerical semigroup and $S=\langle A\rangle$, then we say that $A$ is a system of generators of $S$. In addition, if $S \neq\langle X\rangle$ for all $X \subsetneq A$, then we say that $A$ is a minimal system of generators of $S$. In [24] it is proved that every numerical semigroup admits a unique minimal system of generators. Moreover, such a minimal system of generators is finite. In this way, the cardinality of the minimal system of generators of $S$ is called the embedding dimension of $S$ and denoted by e $(S)$.

Since $\mathbb{N} \backslash S$ is finite, we can study three notable invariants of $S$. Namely, the maximum integer that does not belong to $S$, which is called the Frobenius number of $S$ and denoted by $\mathrm{F}(S)$; the least positive integer that belongs to $S$, which is called the multiplicity of $S$ and denoted by $\mathrm{m}(S)$; and the cardinality of the set $\mathbb{N} \backslash S$, which is called the genus of $S$ and denoted by $\mathrm{g}(S)$.

The Frobenius problem (see [18]) consists in finding formulas for the Frobenius number and the genus of a numerical semigroup in terms of its minimal system of generators. This problem was solved by Sylvester and Curran Sharp (see [28, 29], 30]) when the embedding dimension is equal to two $(\mathrm{e}(\mathrm{S})=2)$. At present, the Frobenius problem is open for the case e $(S) \geq 3$. Indeed, Curtis showed in 8 , that it is impossible to find a polynomial formula (that is, a finite set of polynomials) that computes the Frobenius number if the embedding dimension equal to three. Moreover, using Turing reductions, Ramírez-Alfonsín proved in [17] that this problem is NP-hard for arbitrary embedding dimension. Despite of this, there exists a long list of papers about this topic.

With Selmer's formulas (see [26]), the computation of an Apéry set (see [2]) solves the Frobenius problem. Let $S$ be a numerical semigroup and $s \in S \backslash\{0\}$. The Apéry set of $s$ in $S$ is $\operatorname{Ap}(S, s)=\{x \in S \mid x-s \notin S\}$. It is known that this set is finite and, in addition, we have the expressions $\mathrm{F}(S)=\max (\operatorname{Ap}(S, s))-s$ and $\mathrm{g}(S)=\frac{1}{s}\left(\sum_{w \in \operatorname{Ap}(S, s) w}\right)-\frac{s-1}{2}$.

\footnotetext{
*Both authors are supported by the project MTM2014-55367-P, which is funded by Ministerio de Economía y Competitividad and Fondo Europeo de Desarrollo Regional FEDER, and by the Junta de Andalucía Grant Number FQM-343. The second author is also partially supported by Junta de Andalucía/Feder Grant Number FQM-5849.

†Departamento de Matemática Aplicada, Universidad de Granada, 18071-Granada, Spain. E-mail: arobles@ugr.es

¥Departamento de Álgebra, Universidad de Granada, 18071-Granada, Spain. E-mail: jrosales@ugr.es
} 
In some cases a recursive approach can be used. Let $\left\{a_{1}, \ldots, a_{n}\right\}$ be the minimal system of generators of a numerical semigroup $S$, with $\operatorname{gcd}\left(\left\{a_{1}, \ldots, a_{n-1}\right\}\right)=d$. If we denote by $S^{\prime}=\left\langle\frac{a_{1}}{d}, \ldots, \frac{a_{n-1}}{d}, a_{n}\right\rangle$, then it is well known (see [7, [13, 20]) that $\mathrm{F}(S)=d \cdot \mathrm{F}\left(S^{\prime}\right)+a_{n}(d-1)$ and $\mathrm{g}(S)=d \cdot \mathrm{g}\left(S^{\prime}\right)+\frac{1}{2}\left(a_{n}-1\right)(d-1)$.

The ideas of the above paragraphs (together with "L-shaped" regions of lattice points, Euclidean algorithms, continued fractions expansions of certain quotients and Hilbert series) are used, among others, by Brauer and Shockley ([6], [7]), Davison ([9]), Djawadi and Hofmeister ([10]), Greenberg ([12]), Johnson ([13]), Kannan ([14), Rodseth and Ramírez Alfonsín (20], 21], [19]), Selmer and Beyer ([26], 27]), in order to solve the Frobenius problem. In most cases, the authors give algorithms and/or lower and upper bounds. Moreover, in some particular settings, exact formulas are provided (see [23]). For more details about these and others works in this line, we refer the reader to the book by Ramírez Alfonsín ([18]) and, in addition, an expository paper by Owens ([16]).

Geometrical tools (in particular, geometric interpretations of continued fractions) were suggested by Arnold (see [3, 4]) for studying the Frobenius problem. Some authors, as Aicardi (see [1]) and Ustinov (see [31]) have used this approach.

Also, Gröbner bases are being used to obtain efficient algorithms to solve the Frobenius problem. Following this via, we should cite the papers by Roune (see [25]), Márquez-Campos, Ojeda, and Tornero (see [15]), and the references therein.

The reader not familiar with the study of numerical semigroups can think that some of the used notation (embedding dimension, genus, multiplicity, ...) is surprising. However, in the literature there are many works devoted to the study of the analytically irreducible one dimensional local domains via their value semigroups, which turn out to be numerical semigroups. All the invariants that are mentioned in this paper have their interpretation in this context, and this is the reason of their names. In this sense, [5] is a very adequate reference in order to translate results between the numerical semigroups theory and the rings theory.

We will denote by $\mathfrak{L}(m, g)$ the set of all numerical semigroups with multiplicity $m$ and genus $g$. Let us observe that, if $S$ is a numerical semigroup and $1 \in S$, then $S=\mathbb{N}$. Therefore, if $\mathfrak{L}(1, g) \neq \emptyset$, then $g=0$ and $\mathfrak{L}(1,0)=\{\mathbb{N}\}$. From now on we will suppose that $m$ is an integer greater than or equal to two. We will begin our work showing that $\mathfrak{L}(m, g) \neq \emptyset$ if and only if $m \leq g+1$. From that point on, the main purpose of this paper is to study the set $\{\mathrm{F}(S) \mid S \in \mathfrak{L}(m, g)\}$.

As usual, if $x$ is a rational number, we set $\lceil x\rceil=\min \{z \in \mathbb{Z} \mid x \leq z\}$. In Section 2 we will show that, if $S \in \mathfrak{L}(m, g)$, then $\mathrm{F}(S) \geq\left\lceil\frac{m g}{m-1}\right\rceil-1$. Moreover, we will see that there exists a unique numerical semigroup that achieves such bound.

Let us observe that, if $m=g+1$, then $\mathfrak{L}(m, g)=\{\{0, g+1, \rightarrow\}\}$ (where the symbol $\rightarrow$ means that every integer greater than $g+1$ belongs to the set). Thus, from now on, we will suppose that $m \leq g$. In Section 3 we will show that, if $S \in \mathfrak{L}(m, g)$, then

- $\mathrm{F}(S) \leq 2 g-1$ if $m \nmid(2 g-1)$ (where, if $a, b$ are positive integers, $a \nmid b$ means that $b$ is not divisible by $a$ ).

- $\mathrm{F}(S) \leq 2 g-2$ if $m \mid(2 g-1)$ (where, if $a, b$ are positive integers, $a \mid b$ means that $a$ divides $b$ ).

In addition, we will see that both of the bounds are achieved, but not necessarily in a unique numerical semigroup.

Finally, in Section 4 we will show that

$$
\{\mathrm{F}(S) \mid S \in \mathfrak{L}(m, g)\}=\left\{F \in \mathbb{N} \mid m \nmid F \text { and }\left\lceil\frac{m g}{m-1}\right\rceil-1 \leq F \leq 2 g-1\right\} .
$$

Before go the next section, we want to notice that, in order to facilitate the searching of some results and facts, together with the original references, we have included the corresponding reference in [24].

\section{A lower bound for the Frobenius number}

Let us observe that, if $S$ is a numerical semigroup, then $\mathrm{m}(S) \in \mathbb{N} \backslash\{0\}$ and $\mathrm{g}(S) \in \mathbb{N}$.

Proposition 2.1. Let $(m, g) \in(\mathbb{N} \backslash\{0\}) \times \mathbb{N}$. Then $\mathfrak{L}(m, g) \neq \emptyset$ if and only if $(m, g)=(1,0)$ or $2 \leq m \leq g+1$.

Proof. (Necessity). If $m=1$ and $S \in \mathfrak{L}(1, g)$, then it is obvious that $S=\mathbb{N}$ and, thereby, $g=0$. Therefore, we can suppose that $2 \leq m$. Now, if $S \in \mathfrak{L}(m, g)$, then $\{1, \ldots, m-1\} \cap S=\emptyset$ and, consequently, $g \geq m-1$.

(Sufficiency). Since $\mathbb{N} \in \mathfrak{L}(1,0)$, we have that $\mathfrak{L}(1,0) \neq \emptyset$. So, let us suppose that $2 \leq m \leq g+1$. Let $q, r$ be two nonnegative integers such that $g=q(m-1)+r$, with $0 \leq r \leq m-2$ (that is, $q$ and $r$ are the 
quotient and the remainder of the division of $g$ by $m-1)$. Let $S=\{0, m, 2 m, \ldots, q m\} \cup\{q m+r+1, \rightarrow\}$. It is clear that $S$ is a numerical semigroup with multiplicity $m$ and that $\operatorname{g}(S)=q(m-1)+r=g$. Therefore, $S \in \mathfrak{L}(m, g)$, that is, $\mathfrak{L}(m, g) \neq \emptyset$.

Our next aim will be to prove Proposition 2.3 . For that we need to introduce some concepts and results.

Following the notation introduced in $[22$, we say that $x \in \mathbb{Z} \backslash S$ is a pseudo-Frobenius number of $S$ if $x+s \in S$ for all $s \in S \backslash\{0\}$. The cardinality of the set consisting of all pseudo-Frobenius numbers of $S$ is an important invariant of $S$ which is called the type of $S$ (see [5] ) and is denoted by t $(S)$.

If $S$ is a numerical semigroup, then we will denote by $\mathrm{n}(S)$ the cardinality of the set $\{s \in S \mid s<\mathrm{F}(S)\}$. It is clear that $\mathrm{g}(S)+\mathrm{n}(S)=\mathrm{F}(S)+1$. On the other hand, we have the following consequence of 11, Corollary 2, Theorem 20] (in alternation, see [24, Corollary 2.23, Proposition 2.26]).

Lemma 2.2. If $S$ is a numerical semigroup, then $\mathrm{g}(S) \leq \mathrm{t}(S) \mathrm{n}(S)$ and $\mathrm{t}(S) \leq \mathrm{m}(S)-1$. Consequently, $\mathrm{g}(S) \leq(\mathrm{m}(S)-1) \mathrm{n}(S)$.

Now we are ready to show the announced result.

Proposition 2.3. If $S$ is a numerical semigroup with $\mathrm{m}(S) \geq 2$, then

$$
\left\lceil\frac{\mathrm{m}(S) \mathrm{g}(S)}{\mathrm{m}(S)-1}\right\rceil-1 \leq \mathrm{F}(S)
$$

Proof. By Lemma 2.2, we know that $\mathrm{g}(S) \leq(\mathrm{m}(S)-1) \mathrm{n}(S)$. Therefore,

$$
\mathrm{m}(S) \mathrm{g}(S) \leq(\mathrm{m}(S)-1)(\mathrm{n}(S)+\mathrm{g}(S))=(\mathrm{m}(S)-1)(\mathrm{F}(S)+1),
$$

and then the inequality follows easily.

In the next result we will see that it is possible to reach the bound of the previous proposition.

Proposition 2.4. Let $m, g$ be integers such that $2 \leq m \leq g+1$. Then there exists $S \in \mathfrak{L}(m, g)$ such that $\mathrm{F}(S)=\left\lceil\frac{m g}{m-1}\right\rceil-1$.

Proof. Let us have $q, r \in \mathbb{N}$ such that $g=q(m-1)+r$, with $0 \leq r \leq m-2$. Beginning with proof of Proposition 2.1, we know that $S=\{0, m, \ldots, q m\} \cup\{q m+r+1, \rightarrow\} \in \mathfrak{L}(m, g)$. In order to finish this proof, let us see that $\mathrm{F}(S)+1=\left\lceil\frac{m g}{g-1}\right\rceil$. For that, since $\left\lceil\frac{m g}{m-1}\right\rceil \leq \mathrm{F}(S)+1$ by Proposition 2.3 , will be enough to show that $\mathrm{F}(S)+1-\frac{m g}{m-1}<1$. Let us observe that

$$
\mathrm{F}(S)=\left\{\begin{array}{l}
q m-1, \text { if } r=0, \\
q m+r, \text { if } r \neq 0,
\end{array}\right.
$$

and $\mathrm{g}(S)=(m-1) q+r$. Thus, we distinguish two cases.

1. If $r=0$, then $\mathrm{F}(S)+1-\frac{m g}{m-1}=q m-\frac{m q(m-1)}{m-1}=0<1$.

2. If $r \neq 0$, then $\mathrm{F}(S)+1-\frac{m g}{m-1}=q m+r+1-\frac{m(q(m-1)+r)}{m-1}=\frac{m-1-r}{m-1}<1$.

We finish this section by showing that the semigroup of $\mathfrak{L}(m, g)$ which reaches the bound of Proposition 2.3 is unique.

Proposition 2.5. Let $m, g$ be integers such that $2 \leq m \leq g+1$. If $S \in \mathfrak{L}(m, g)$ and $\mathrm{F}(S)=\left\lceil\frac{m g}{m-1}\right\rceil-1$, then $S=\{0, m, \ldots, q m\} \cup\{q m+r+1, \rightarrow\}$, where $q, r$ are the unique non-negative integers such that $g=q(m-1)+r$ with $0 \leq r \leq m-2$.

Proof. Let $T=\{0, m, \ldots, q m\} \cup\{q m+r+1, \rightarrow\}$. From proof of Proposition 2.4 we know that $T$ is a numerical semigroup with genus equal to $g$ and such that $\mathrm{F}(T)=\left\lceil\frac{m g}{m-1}\right\rceil-1$. On the other hand, it is clear that, if $S \in \mathfrak{L}(m, g)$ and $\mathrm{F}(S)+1=\left\lceil\frac{m g}{m-1}\right\rceil$, then $T \subseteq S$. Since $\mathrm{g}(T)=g=\mathrm{g}(S)$, we conclude that $S=T$. 


\section{An upper bound for the Frobenius number}

Now we want to give the upper bound of the set $\{\mathrm{F}(S) \mid S \in \mathfrak{L}(m, g)\}$. In contrast with the given lower bound in the previous section, we will show that the upper bound "almost" does not depend of $m$.

The following result is [24, Lemma 2.14].

Lemma 3.1. If $S$ is a numerical semigroup, then $\mathrm{F}(S) \leq 2 \mathrm{~g}(S)-1$.

Proposition 3.2. Let $m, g$ be integers such that $2 \leq m \leq g+1$. If $S \in \mathfrak{L}(m, g)$, then

1. $\mathrm{F}(S)=g$ if $m=g+1$.

2. $\mathrm{F}(S) \leq 2 g-1$ if $m \leq g$ and $m \nmid(2 g-1)$.

3. $\mathrm{F}(S) \leq 2 g-2$ if $m \leq g$ and $m \mid(2 g-1)$.

Proof. 1. Evident.

2. It is an immediate consequence of Lemma 3.1.

3. By Lemma 3.1, it is sufficient to show that $\mathrm{F}(S) \neq 2 g-1$. And this is clear because, if $\mathrm{F}(S)=2 g-1$, then $2 g-1 \notin S$, in contradiction with the fact that $m \in S$ and $m \mid(2 g-1)$.

Our next goal will be show that the bounds of the previous proposition are reached. For that, we introduce several concepts and results.

A numerical semigroup is irreducible if it cannot be expressed as the intersection of two numerical semigroups properly containing it. Irreducible numerical semigroups with odd (even, respectively) Frobenius number are called symmetric (pseudo-symmetric, respectively). From [11, Lemma 1, Lemma 3], we have the following result (alternatively see [24, Corollary 4.5]).

Lemma 3.3. Let $S$ be a numerical semigroup.

1. $S$ is symmetric if and only if $\mathrm{g}(S)=\frac{\mathrm{F}(S)+1}{2}$.

2. $S$ is pseudo-symmetric if and only if $\mathrm{g}(S)=\frac{\mathrm{F}(S)+2}{2}$.

By [24, Theorem 4.2, Lemma 4.3, Proposition 4.4], we have the next lemma.

Lemma 3.4. Let $\mathfrak{L}(F)$ be the set of all numerical semigroups with Frobenius number equal to $F$. If $S \in \mathfrak{L}(F)$, then

1. $S$ is irreducible if and only if $S$ is maximal (with respect the set inclusion) in $\mathfrak{L}(F)$.

2. If $h=\max \left\{x \in \mathbb{N} \backslash S \mid F-x \notin S\right.$ and $\left.x \neq \frac{F}{2}\right\}$, then $S \cup\{h\} \in \mathfrak{L}(F)$.

3. $S$ is irreducible if and only if $\left\{x \in \mathbb{N} \backslash S \mid F-x \notin S\right.$ and $\left.x \neq \frac{F}{2}\right\}=\emptyset$.

4. $S \cup\left\{x \in \mathbb{N} \backslash S \mid F-x \notin S\right.$ and $\left.x>\frac{F}{2}\right\}$ is an irreducible numerical semigroup with Frobenius number equal to $F$.

Remark 3.5. The first assert of the above lemma is a direct consequence of [11, Proposition 4].

Proposition 3.6. Let $m, g$ be integers such that $2 \leq m \leq g$.

1. If $m \nmid(2 g-1)$, then there exists $S \in \mathfrak{L}(m, g)$ such that $\mathrm{F}(S)=2 g-1$.

2. If $m \mid(2 g-1)$, then there exists $S \in \mathfrak{L}(m, g)$ such that $\mathrm{F}(S)=2 g-2$.

Proof. 1. It is clear that $\bar{S}=\langle m\rangle \cup\{2 g, \rightarrow\}$ is a numerical semigroup with $\mathrm{m}(\bar{S})=m$ and $\mathrm{F}(\bar{S})=2 g-1$. By Item 4 of Lemma 3.4, we have that $S=\bar{S} \cup\left\{x \in \mathbb{N} \backslash \bar{S} \mid \mathrm{F}(\bar{S})-x \notin \bar{S}\right.$ and $\left.x>\frac{\mathrm{F}(\bar{S})}{2}\right\}$ is a symmetric numerical semigroup with $\mathrm{F}(S)=2 g-1$. Let us observe that, if $x$ is an integer greater than $\frac{\mathrm{F}(\bar{S})}{2}$, then $x \geq\left\lceil\frac{2 g-1}{2}\right\rceil=g \geq m$. Thereby, $\mathrm{m}(S)=m$. Moreover, by Lemma 3.3 , we have that $\mathrm{g}(S)=\frac{\mathrm{F}\left(S^{2}\right)+1}{2}=g$. Therefore, $S \in \mathfrak{L}(m, g)$ and $\mathrm{F}(S)=2 g-1$. 
2. It is clear that $\bar{S}=\langle m\rangle \cup\{2 g-1, \rightarrow\}$ is a numerical semigroup with $\mathrm{m}(\bar{S})=m$ and $\mathrm{F}(\bar{S})=2 g-2$ (let us observe that, if $m \mid(2 g-1)$, then $m \nmid(2 g-2))$. Following the same reasoning as in the previous item, the proof is complete.

We will finish this section with several examples in which the numerical semigroups, that reach the given bounds by Items 2 and 3 of Proposition 3.2 , are not unique.

Example 3.7. It is easy to check that $\langle 5,6,9\rangle,\langle 5,7,9,11\rangle \in \mathfrak{L}(5,7)$. Moreover, $5 \nmid(2 \times 7-1)$ and $\mathrm{F}(\langle 5,6,9\rangle)=$ $\mathrm{F}(\langle 5,7,9,11\rangle)=2 \times 7-1=13$.

Example 3.8. It is clear that $\langle 7,8,25\rangle,\langle 7,11,19,24\rangle \in \mathfrak{L}(7,18)$. Moreover, $7 \mid(2 \times 18-1)$ and $\mathrm{F}(\langle 7,8,25\rangle)=$ $\mathrm{F}(\langle 7,11,19,24\rangle)=2 \times 18-2=34$.

\section{Variability of the Frobenius number}

As a consequence of Propositions 2.3 and 3.2 , we know that, if $S \in \mathfrak{L}(m, g)$, then $\left\lceil\frac{m g}{m-1}\right\rceil-1 \leq \mathrm{F}(S) \leq 2 g-1$. Moreover, let us observe that, if $S \in \mathfrak{L}(m, g)$, then $m \nmid \mathrm{F}(S)$. In this section, our aim will be to prove that

$$
\{\mathrm{F}(S) \mid S \in \mathfrak{L}(m, g)\}=\left\{F \in \mathbb{N} \mid m \nmid F \text { and }\left\lceil\frac{m g}{m-1}\right\rceil-1 \leq F \leq 2 g-1\right\} .
$$

Lemma 4.1. Let $S, \bar{S}$ be numerical semigroups such that $S \subseteq \bar{S}$. Let us suppose that $\bar{S} \backslash S=\left\{x_{1}, \ldots, x_{r}\right\}$ with $x_{1}>\cdots>x_{r}$. Then $S \cup\left\{x_{1}, \ldots, x_{i}\right\}$ is a numerical semigroup for each $i \in\{1, \ldots, r\}$.

Proof. It is enough to observe that the sum of two elements of $\left\{x_{1}, \ldots, x_{i}\right\}$, or the sum of an element of $S$ and an element of $\left\{x_{1}, \ldots, x_{i}\right\}$, is another element of $\bar{S}$ that is greater than or equal to $x_{i}$. Therefore, this another element belongs to $S \cup\left\{x_{1}, \ldots, x_{i}\right\}$.

If $x$ is a rational number, then we will denote $\lfloor x\rfloor=\max \{z \in \mathbb{Z} \mid z \leq x\}$. On the other hand, if $a, b$ are positive integers, then we will denote $a \bmod b=a-\left\lfloor\frac{a}{b}\right\rfloor b$. Let us observe that $a=\left\lfloor\frac{a}{b}\right\rfloor b+a$ mod $b$, and that $a \bmod b \in\{0, \ldots, b-1\}$.

Lemma 4.2. Let $m, F, g$ be positive integers. Then $F-\left\lfloor\frac{F}{m}\right\rfloor \geq g$ if and only if $F+1 \geq\left\lceil\frac{m g}{m-1}\right\rceil$.

Proof. (Necessity). If $F-\left\lfloor\frac{F}{m}\right\rfloor \geq g$, then $F m-\left\lfloor\frac{F}{m}\right\rfloor m \geq g m$. Thus, $F m-(F-F \bmod m) \geq g m$, and, thereby, $F \geq \frac{m g}{m-1}-\frac{F \bmod m}{m-1} \geq \frac{m g}{m-1}-1$. Therefore, $F+1 \geq\left\lceil\frac{m g}{m-1}\right\rceil$.

(Sufficiency). If $F+1 \geq\left\lceil\frac{m g}{m-1}\right\rceil$, then $F+1 \geq \frac{m g}{m-1}$, that is, $F m-F+m-1 \geq m g$. Consequently, $F-\left\lfloor\frac{F}{m}\right\rfloor+\frac{m-1-F \bmod m}{m} \geq g$. Since $F-\left\lfloor\frac{F}{m}\right\rfloor$ and $g$ are integers, and $0 \leq \frac{m-1-F \bmod m}{m}<1$, we conclude that $F-\left\lfloor\frac{F}{m}\right\rfloor \geq g$.

Now, we are prepared to prove the result that we announced at the beginning of this section.

Theorem 4.3. Let $m, g, F$ positive integers such that $2 \leq m \leq g$ and $m \nmid F$. Then there exists $S \in \mathfrak{L}(m, g)$ such that $\mathrm{F}(S)=F$ if and only if $\left\lceil\frac{m g}{m-1}\right\rceil-1 \leq F \leq 2 g-1$.

Proof. (Necessity). It is an immediate consequence of Propositions 2.3 and 3.2 .

(Sufficiency). Depending on $m<\frac{F}{2}$ or $m>\frac{F}{2}$, we distinguish two cases.

1. Let us suppose that $m<\frac{F}{2}$. Let $S=\left\{0, m, \ldots,\left\lfloor\frac{F}{m}\right\rfloor m\right\} \cup\{F+1, \rightarrow\}$. It is clear that $S$ is a numerical semigroup with multiplicity $m$ and Frobenius number $F$. Moreover, $\mathrm{g}(S)=F-\left\lfloor\frac{F}{m}\right\rfloor$. Since $F+1 \geq\left\lceil\frac{m g}{m-1}\right\rceil$, by Lemma 4.2 , then we have that $\mathrm{g}(S) \geq g$. From Item 4 of Lemma 3.4 , there exist integers $x_{1}, x_{2}, \ldots, x_{r}$ such that $x_{1}>x_{2}>\cdots>x_{r}>\frac{F}{2}>m$ and $\bar{S}=S \cup\left\{x_{1}, \ldots, x_{r}\right\}$ is an irreducible numerical semigroup with Frobenius number $F$. In addition, it is clear that $\mathrm{m}(\bar{S})=m$ and, by Lemma 3.3 , that $\mathrm{g}(\bar{S})=\left\lceil\frac{F+1}{2}\right\rceil$. Now, let us observe that $\mathrm{g}(S) \geq g \geq\left\lceil\frac{F+1}{2}\right\rceil=\mathrm{g}(\bar{S})$. Applying Lemma 4.1 we have that $S^{\prime}=S \cup\left\{x_{1}, \ldots, x_{\mathrm{g}(S)-g}\right\}$ is a numerical semigroup. Moreover, we have easily that $\mathrm{m}\left(S^{\prime}\right)=m$ and $\mathrm{F}\left(S^{\prime}\right)=F$. Finally, $\mathrm{g}\left(S^{\prime}\right)=\mathrm{g}(S)-(\mathrm{g}(S)-g)=g$. 
2. Let us suppose that $m>\frac{F}{2}$. The set $\{m, \ldots, F-1\}$ has cardinality $F-m$. Since $m \leq g$, then $F-m \geq F-g$. Therefore, we can take a subset $A \subseteq\{m, \ldots, F-1\}$ such that $m \in A$ and the cardinality of $A$ is equal to $F-g$. Being that all the elements of $A$ are greater that $\frac{F}{2}$, then it is easy to deduce that $S=A \cup\{F+1, \rightarrow\} \cup\{0\} \in \mathfrak{L}(m, g)$ and $\mathrm{F}(S)=F$.

\section{References}

[1] F. Aicardi, On the geometry of the Frobenius problem, Funct. Anal. Other Math. 2 (2009), 111-127.

[2] R. Apéry, Sur les branches superlinéaires des courbes algébriques, C. R. Acad. Sci. Paris 222 (1946), $1198-1200$.

[3] V. I. Arnold, Weak asymptotics for the numbers of solutions of Diophantine problems, Functional Analysis and its Applications 33(4) (1999), 292-293.

[4] V. I. Arnold, Geometry of continued fractions associated with Frobenius numbers, Funct. Anal. Other Math. 2 (2009), 129-138.

[5] V. Barucci, D. E. Dobbs, and M. Fontana, Maximality Properties in Numerical Semigroups and Applications to One-Dimensional Analytically Irreducible Local Domains, Mem. Amer. Math. Soc., Vol. 125, no. 598 (American Mathematical Society, Providence, 1997).

[6] A. Brauer, On a problem of partitions, Amer. J. Math. 64 (1942), 299-312.

[7] A. Brauer, J. E. Shockley, On a problem of Frobenius, J. Reine Angew. Math. 211 (1962), 215-220.

[8] F. Curtis, On formulas for the Frobenius number of a numerical semigroup, Math. Scand. 67 (1990), 190-192.

[9] J. L. Davison, On the linear Diophantie problem of Frobenius, J. Number Theory 48 (1994), 353-363.

[10] M. Djawadi, G. Hofmeister, Linear Diophantine problems, Arch. Math. (Basel) 66 (1996), 19-29.

[11] R. Fröberg, C. Gottlieb, and R. Häggkvist, On numerical semigroups, Semigroup Forum 35 (1987), $63-83$.

[12] H. Greenberg, Solution to a linear diophantine equation for nonnegative integers, Journal of Algorithms 9 (1988), 343-353.

[13] S. M. Johnson, A linear Diophantine problem, Can. J. Math. 12 (1960), 390-398.

[14] R. Kannan, Lattice translates of a polytope and the Frobenius problem, Combinatorica 12 (1992), 161177.

[15] G. Márquez-Campos, I. Ojeda, J. M. Tornero, On the computation of the Apéry set of numerical monoids and affine semigroups, Semigroup Forum 91 (2015), 139-158.

[16] R. W. Owens, An algorithm to solve the Frobenius problem, Mathematics Magazine 76(4) (2003), 264275 .

[17] J. L. Ramírez-Alfonsín, Complexity of the Frobenius problem, Combinatorica 16 (1996), 143-147.

[18] J. L. Ramírez Alfonsín, The Diophantine Frobenius Problem, Oxford Lectures Series in Mathematics and its Applications 30 (Oxford University Press, Oxford, 2005).

[19] J. L. Ramírez Alfonsín, Ø. J. Rødseth, Numerical semigroups: Apéry sets and Hilberts series, Semigroup Forum 79 (2009), 323-340.

[20] Ö. J. Rödseth, On a linear Diophantine problem of Frobenius, J. Reine Angew. Math. 301 (1978), $171-178$. 
[21] Ö. J. Rödseth, On a linear Diophantine problem of Frobenius II, J. Reine Angew. Math. 307/308 (1979), 431-440.

[22] J. C. Rosales and M. B. Branco, Numerical semigroups that can be expressed as an intersection of symmetric numerical semigroups, J. Pure Appl Algebra 171 (2002), 303-314.

[23] J. C. Rosales and P. A. García-Sánchez, Pseudo-symmetric numerical semigroups with three generators, J. Algebra 291 (2005), 46-54.

[24] J. C. Rosales and P. A. García-Sánchez, Numerical semigroups, Developments in Mathematics, Vol. 20 (Springer, New York, 2009).

[25] B. H. Roune, Solving thousand-digit Frobenius problems using Gröbner bases, J. Symbolic Comput. 43 (2008), 1-7.

[26] E. S. Selmer, On the linear diophantine problem of Frobenius J. Reine Angew. Math. 293/294 (1977), $1-17$.

[27] E. S. Selmer and O. Beyer, On the linear diophantine problem of Frobenius in three variables, J. Reine Angew. Math. 301 (1978), 161-170.

[28] J. J. Sylvester, On subvariants, i.e. semi-invariants to binary quantics of an unlimited order, Amer. J. Math. 5 (1882), 79-136.

[29] J. J. Sylvester, Problem 7382, The Educational Times, and Journal of the College of Preceptors, New Series 36(266) (1883), 177. Solution by W. J. Curran Sharp, ibid., 36(271) (1883), 315. Republished as 30.

[30] J. J. Sylvester, Problem 7382, in W. J. C. Miller (Ed.), Mathematical questions, with their solutions, from the "Educational Times", Vol. 41, page 21 (Francis Hodgson, London, 1884).

[31] A. V. Ustinov, Geometric proof of Rødseth's formula for Frobenius numbers, Proocedings of the Steklov Institute of Mathematics 276 (2012), 275-282. 